\title{
Multimode Electron Tomography as a Tool to Characterize the Internal Structure and Morphology of Gold Nanoparticles
}

\author{
Naomi Winckelmans, ${ }^{\dagger}$ Thomas Altantzis, ${ }^{\dagger}$ Marek Grzelczak, $^{\ddagger}, \S_{\odot}$ Ana Sánchez-Iglesias, ${ }^{\ddagger}$ \\ Luis M. Liz-Marzán, ${ }^{\ddagger},{ }^{\circ}$ and Sara Bals ${ }^{*}{ }^{\dagger}$ (i) \\ ${ }^{\dagger}$ EMAT-University of Antwerp, Groenenborgerlaan 171, B-2020 Antwerp, Belgium \\ ${ }^{\ddagger}$ CIC biomaGUNE and CIBER-BBN, Paseo de Miramón 182, 20014 Donostia-San Sebastián, Spain \\ ${ }^{\S}$ Ikerbasque, Basque Foundation for Science, 48013 Bilbao, Spain
}

\section{Supporting Information}

\begin{abstract}
Three dimensional (3D) characterization of structural defects in nanoparticles by transmission electron microscopy is far from straightforward. We propose the use of a dose-efficient approach, so-called multimode tomography, during which tilt series of low and high angle annular dark field scanning transmission electron microscopy projection images are acquired simultaneously. In this manner, not only reliable information can be obtained concerning the shape of the nanoparticles, but also the twin planes can be clearly visualized in $3 \mathrm{D}$. As an example, we demonstrate the application of this approach to identify the position of the seeds with respect to the twinning planes in anisotropic gold nanoparticles synthesized using a seed mediated growth approach.
\end{abstract}

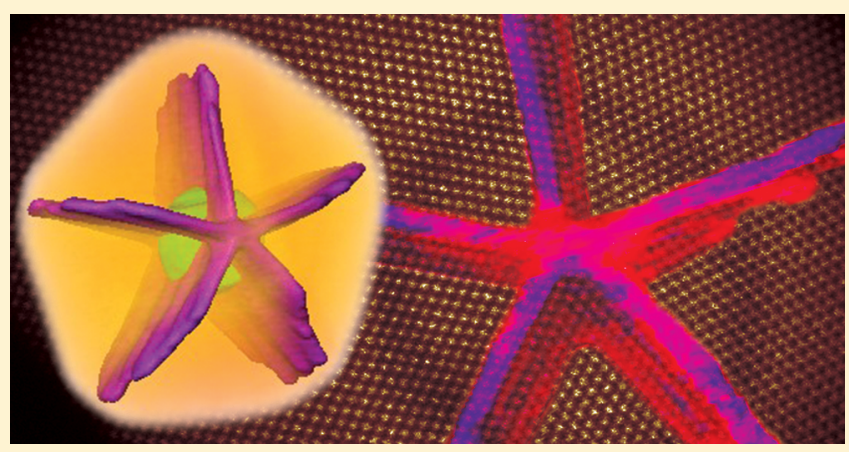

\section{INTRODUCTION}

Gold nanoparticles are of great interest due to their unique optical properties, which render them promising materials for a variety of applications such as surface-enhanced Raman scattering, plasmonics, and biosensing. ${ }^{1-3}$ Since the optical properties of plasmonic nanomaterials heavily depend on their morphology, accurate and reproducible synthetic methods are of primary importance. In this context, the three-dimensional (3D) structural characterization of such particles is an essential tool toward understanding the mechanisms involved in metal nanoparticle growth. ${ }^{4-7}$ Transmission electron microscopy (TEM) and scanning transmission electron microscopy (STEM) have become standard techniques to investigate the structure of nanomaterials. However, these techniques only provide a two-dimensional (2D) projection of objects with a $3 \mathrm{D}$ structure, which often displays a more complex structure than it appears. When investigating the structure of nanoparticles in $3 \mathrm{D}$, electron tomography is required. Hereby, a tilt series of projection images is acquired over an as wide as possible angular range. Next, these images serve as an input for a mathematical reconstruction algorithm that enables one to reconstruct the $3 \mathrm{D}$ structure of the nanoparticle of interest. Since the turn of the century, electron tomography has been used to investigate a broad range of nanomaterials. ${ }^{8-10}$ The increasing complexity of nanomaterials has driven the development of even more sophisticated characterization techniques in which tomography is combined with, for example, energy dispersive X-ray (EDS) spectroscopy ${ }^{11,12}$ and electron energy loss spectroscopy (EELS). ${ }^{13}$ In this manner, the $3 \mathrm{D}$ investigation of the morphology, composition, and oxidation states in nanomaterials has become possible.

Not only the morphology, also structural defects can have a huge impact on the properties of nanomaterials, as they directly affect plasmonic or catalytic properties. ${ }^{14-16}$ For example, screw dislocations can enhance the transport of molecules in nanoporous zeolites ${ }^{17}$ and twin domains can enhance the photocatalytic activity in ternary chalcogenate nanorods. ${ }^{18}$ Furthermore, in Au nanoparticles, which are often synthesized using a seed mediated approach, ${ }^{19,20}$ the presence of twins in the seed can be exploited to obtain nanoparticles with morphologies that would otherwise not be feasible. ${ }^{21-23}$ The ability to accurately visualize defects in $3 \mathrm{D}$ is therefore of great relevance. At the atomic scale, 3D visualization of atomic defects has been demonstrated using various approaches. ${ }^{24,25}$ However, such studies are still challenging and mostly restricted to particles with sizes below $20 \mathrm{~nm}$. At the nanometer level, several electron microscopy methods have been developed to visualize crystalline defects in $3 \mathrm{D}$, including tomography based on weak beam electron microscopy, ${ }^{26,27}$ medium angle annular dark field scanning transmission electron microscopy (MAADF-STEM), ${ }^{27,28}$ and scanning electron diffraction

Special Issue: Prashant V. Kamat Festschrift

Received: December 16, 2017

Revised: January 16, 2018

Published: January 16, 2018 
(SED). ${ }^{29}$ For such experiments, the main challenge is that the projection requirement for tomography needs to be fulfilled, stating that the intensity of the acquired images should be a monotonic function of a certain property of the sample under investigation. ${ }^{8}$ Electron tomography studies are therefore mostly based on high angle annular dark field scanning transmission electron microscopy (HAADF-STEM), since the image intensity in this technique scales with the atomic number $\mathrm{Z}$ of the elements under investigation and the thickness of the sample. $^{30}$ However, when using this technique, diffraction contrast, which typically yields information on the presence of defects, is lost. When using MAADF-STEM, the collection angle of the detector is therefore adjusted in such a way that both coherent and incoherent scattered electrons are collected, yielding information about both the shape of the particle and the presence of defects, simultaneously. In the past, this approach has been used to reconstruct dislocations in $3 \mathrm{D},{ }^{27,28}$ but we demonstrate here that a reliable $3 \mathrm{D}$ characterization of twin planes in $\mathrm{Au}$ nanoparticles is hampered by the low contrast of the twins in $2 \mathrm{D}$ projection images. Further decreasing the collection angle of the detector leads to socalled low angle annular dark field STEM (LAADF-STEM). This technique is advantageous to highlight the presence of defects, but since less incoherently scattered electrons are collected, the projection requirement is violated, leading to a lower quality reconstruction of the morphology of the investigated object. It is therefore clear that when selecting an optimal value for the collection angle, one will always have to compromise between optimal contrast to visualize defects and fulfilment of the projection requirement.

In this work, we exploited the flexibility of modern TEM instruments, in which more than one (HA)ADF detector is available. Through the simultaneous use of multiple $\mathrm{ADF}$ detectors, a reliable $3 \mathrm{D}$ reconstruction of both the morphology of the nanoparticles and the twin planes can be achieved in a dose-efficient manner. To demonstrate the applicability and the great potential of this multimode approach, we investigated pentatwinned $\mathrm{Au}$ nanoparticles with different shapes (decahedra, rods, and bipyramids), grown from pentatwinned $\mathrm{Au}$ seeds. ${ }^{31}$ In order to assist finding the position of the seeds in the final particles, gold seeds were covered by a thin layer of $\mathrm{Pd}$, so that the difference in atomic number between $\mathrm{Au}$ and $\mathrm{Pd}$ $\left(Z_{\mathrm{Pd}}=46, Z_{\mathrm{Au}}=79\right)$ enabled us to distinguish between the seed and the grown Au shell using HAADF-STEM. Through this multimode methodology, the position of the seed with respect to the twins could be determined, which provides initial evidence suggesting that bipyramids grown from a core/shell $\mathrm{Au} / \mathrm{Pd}$ seed undergo a different growth mechanism in comparison to bipyramids grown from a pure $\mathrm{Pd}$ seed.

\section{METHODS}

Chemicals. Hexadecyltrimethylammonium chloride (CTAC, 25 wt \% in water), gold(III) chloride trihydrate $\left(\mathrm{HAuCl}_{4}, \geq 99 \%\right)$, citric acid $(\geq 99.5 \%)$, sodium borohydride $\left(\mathrm{NaBH}_{4}\right)$, hexadecyltrimethylammonium bromide (CTAB, $\geq 99 \%)$, silver nitrate $\left(\mathrm{AgNO}_{3}, \geq 99 \%\right)$, hydrochloric acid ( $\mathrm{HCl}, 37 \%)$, L-ascorbic acid (AA, $\geq 99 \%$ ), benzyldimethylhexadecylammonium chloride (BDAC), and potassium tetrachloropalladate(II) $\left(\mathrm{K}_{2} \mathrm{PdCl}_{4}, 99.99 \%\right)$ were purchased from Sigma-Aldrich. All chemicals were used further putification. Milli-Q water (resistivity $18.2 \mathrm{M} \Omega \cdot \mathrm{cm}$ at $25^{\circ} \mathrm{C}$ ) was used in all experiments.
Synthesis of Seeds Solution and Thermal Treatment. The gold seed solution was prepared in a scintillation vial $(20 \mathrm{~mL})$ by rapidly injection of fresh $\mathrm{NaBH}_{4}(0.25 \mathrm{~mL}, 25 \mathrm{mM})$ into the mixture of $\mathrm{HAuCl}_{4}(10 \mathrm{~mL}, 0.25 \mathrm{mM})$ and citric acid $(0.05 \mathrm{~mL}$, $1 \mathrm{M})$ prepared in an aqueous CTAC solution $(50 \mathrm{mM})$ under vigorous stirring at room temperature. The solution turns from light yellow to brownish, indicating the formation of gold seeds. After $2 \mathrm{~min}$, the vial was closed and the solution was heated in an oil bath at $80{ }^{\circ} \mathrm{C}$ during $90 \mathrm{~min}$ under gently stirring to induce twin formation. Aging process led to a gradual color change from brown to red. Thermally treated seed solution was removed from the bath and stored at room temperature. The final concentration of metallic gold was $0.25 \mathrm{mM}$.

Pure Pd seeds were prepared exactly at the same experimental conditions as $\mathrm{Au}$ seeds, but using $\mathrm{K}_{2} \mathrm{PdCl}_{4}$ as precursor.

Synthesis of Palladium-Coated Gold Seeds. To a dispersion of thermally treated gold seeds $(5 \mathrm{~mL}, 0.25 \mathrm{mM})$ was added $\mathrm{K}_{2} \mathrm{PdCl}_{4}(0.025 \mathrm{~mL}$ for $\mathrm{AuPd} 10 \%, 0.050 \mathrm{~mL}$ for AuPd20\%, and $0.125 \mathrm{~mL}$ for AuPd50\%, $5 \mathrm{mM}$ ), and the mixture was left for $15 \mathrm{~min}$ at $40{ }^{\circ} \mathrm{C}$ to allow for complexation of the palladium salt with $\mathrm{CTAB}$, followed by addition of $\mathrm{AA}$ ( $0.025 \mathrm{~mL}$ for AuPd10\%, $0.050 \mathrm{~mL}$ for AuPd20\%, and 0.125 $\mathrm{mL}$ for AuPd50\%, $100 \mathrm{mM}$ ) maintaining the mixture at $40{ }^{\circ} \mathrm{C}$ for $12 \mathrm{~h}$. These seed solutions were used to grow gold bipyramids, pentawinned nanorods, and decahedra.

Synthesis of Pentatwinned Gold Nanoparticles. Gold bipyramids, pentatwinned gold nanorods, and gold decahedra were prepared by a previously reported method. ${ }^{31}$ Synthesis of gold bipyramids: Seeds solution of $\mathrm{Au}, \mathrm{AuPd} 10 \%, \mathrm{AuPd} 20 \%$, or AuPd50\% (1 mL) was added under vigorous stirring to an aqueous growth solution of CTAB $(10 \mathrm{~mL}, 100 \mathrm{mM}), \mathrm{HAuCl}_{4}$ (0.5 mL, $10 \mathrm{mM}), \mathrm{AgNO}_{3}(0.1 \mathrm{~mL}, 10 \mathrm{mM}), \mathrm{HCl}(0.2 \mathrm{~mL}, 1$ $\mathrm{M})$, and $\mathrm{AA}(0.08 \mathrm{~mL}, 100 \mathrm{mM})$. The mixture was left undisturbed at $30{ }^{\circ} \mathrm{C}$ for $2 \mathrm{~h}$. The solution was centrifuged twice $(8000 \mathrm{rpm}, 30 \mathrm{~min})$ to remove excess reactants and dispersed in water.

Synthesis of Pentatwinned Gold Nanorods. An aqueous growth solution of CTAB $(100 \mathrm{~mL}, 8 \mathrm{mM})$ and $\mathrm{HAuCl}_{4}(0.25$ $\mathrm{mL}, 50 \mathrm{mM}$ ) was stirring and cooled down to $20{ }^{\circ} \mathrm{C}$ in a thermostatic bath. After $15 \mathrm{~min}$, an aliquot solution of AA (0.25 $\mathrm{mL}, 100 \mathrm{mM}$ ) was added to the mixture, and the solution was shaken manually until it turns colorless. Subsequently, a certain volume of AuPd10\% solution $(1 \mathrm{~mL})$ was added to the growth solution under vigorous shaken and left undisturbed overnight at $20^{\circ} \mathrm{C}$. The solution was centrifuged $(8000 \mathrm{rpm}, 30 \mathrm{~min})$ to remove excess reactants and dispersed in an aqueous $\mathrm{CTAB}$ solution $(10 \mathrm{~mL}, 1 \mathrm{mM})$, the solution was centrifuged again and finally, dispersed in water.

Synthesis of Gold Decahedra. The solution of $\mathrm{Au}$ or AuPd $10 \%$ seeds $(0.56 \mathrm{~mL})$ was added under vigorous stirring to an aqueous growth solution of BDAC $(10 \mathrm{~mL}, 100 \mathrm{mM})$, $\mathrm{HAuCl}_{4}(0.1 \mathrm{~mL}, 50 \mathrm{mM})$, and $\mathrm{AA}(0.075 \mathrm{~mL}, 100 \mathrm{mM})$ at 30 ${ }^{\circ} \mathrm{C}$. The mixture was left undisturbed at $30{ }^{\circ} \mathrm{C}$ for $30 \mathrm{~min}$. The solution was centrifuged twice $(9000 \mathrm{rpm}, 30 \mathrm{~min})$ to remove excess reactants and dispersed in water.

Characterization. Scanning transmission electron microscopy (STEM) images were obtained with an aberration corrected "cubed" FEI Titan 60-300 electron microscope operated at $300 \mathrm{kV}$. TEM images were obtained with a JEOL JEM-1400PLUS transmission electron microscope operating at an acceleration voltage of $120 \mathrm{kV}$. Samples were prepared by dripping the corresponding nanocrystal solution on carbon- 
coated copper grids. UV-Vis optical extinction spectra were recorded using an Agilent $8453 \mathrm{UV}$-vis diode-array spectrophotometer. Acquisition time for EDX measurements was $\sim 600 \mathrm{~s}$.

\section{RESULTS AND DISCUSSION}

Combination of HAADF-STEM and LAADF-STEM Tomography. Modern TEM columns are equipped with multiple STEM detectors yielding different inner semicollection angles. In practice, the collection angle can be tuned within a specific range by changing the camera length. In Figure 1, we
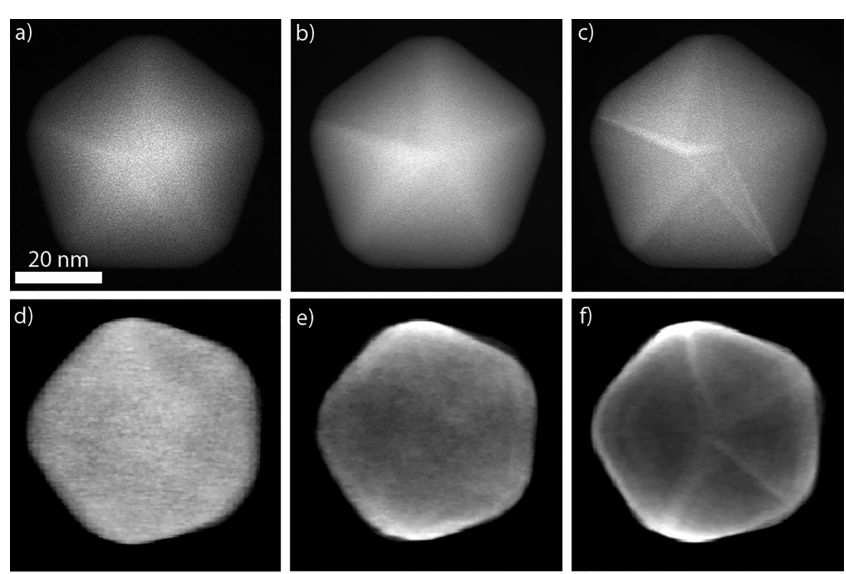

Figure 1. Representative $2 \mathrm{D}$ images and orthoslices through the tomography reconstruction of a Au decahedron, from images acquired in HAADF-STEM (a, d), MAADF-STEM (b, e), and LAADF-STEM (c, f) modes. The inner semicollection angles of the different annular detectors were 115,26 , and $13 \mathrm{mrad}$, respectively.

show representative images of the same pentatwinned $\mathrm{Au}$ decahedron ( $49.6 \pm 0.6 \mathrm{~nm})$, imaged by HAADF-STEM, MAADF-STEM, and LAADF-STEM. Hereby, inner/outer semicollection angles of $115 / 157 \mathrm{mrad}, 26 / 95 \mathrm{mrad}$, and $13 /$ $26 \mathrm{mrad}$ were used, respectively. A schematic diagram of the setup is provided as Supporting Information, Figure S1. In HAADF-STEM mode (Figure 1a), the projected shape of the nanoparticle is clearly visualized, but diffraction contrast related to the presence of twins can hardly be observed. The MAADFSTEM image in Figure $1 \mathrm{~b}$ yields information on the shape, whereas the presence of defects is clearer in comparison to Figure 1a. However, the LAADF-STEM image (Figure 1c) yields the best contrast related to the pentatwinned structure.

Next, tomography series were acquired using the 3 different $\mathrm{ADF}$ detectors, over a tilt range from $-70^{\circ}$ to $+72^{\circ}$ with a tilt increment of $2^{\circ}$. During the acquisition, the image intensities were all scaled between 10000 and 50000 counts per pixel. ${ }^{32}$ The projection images were aligned by a combination of crosscorrelation and a manual tilt axis adjustment as implemented in the FEI Inspect3D software. ${ }^{33}$ For the $3 \mathrm{D}$ reconstruction, the simultaneous iterative reconstruction technique (SIRT) was used as implemented in the ASTRA toolbox. ${ }^{33-35}$ From the orthoslices obtained through the reconstructions, it is clear that the HAADF-STEM reconstruction (Figure 1d) does not yield any contrast corresponding to the twin planes. Limited diffraction contrast can be observed in the MAADF-STEM reconstruction (Figure 1e), whereas the twin planes are evident in the LAADF-STEM reconstruction (Figure 1f). However, the diffraction contrast present in the MAADF-STEM and LAADFSTEM signals violates the projection requirement for tomography. ${ }^{8}$ To investigate the implications of such a violation, we calculated the shape error of these reconstructions using the HAADF-STEM reconstruction as the ground truth morphology. The shape error corresponds to the number of voxels that are labeled differently in the segmentation of the partially coherent reconstructions (MAADF-STEM and LAADF-STEM), as compared to the segmentation of the HAADF-STEM reconstruction. Since the intensities in the acquired images of all series are scaled in the same range, the same threshold could be used for segmentation. Next, the number of misinterpreted voxels is divided by the total number of nonzero voxels in the HAADF-STEM reconstruction. ${ }^{36}$ For the MAADF-STEM reconstruction, a shape error of $4.4 \%$ was found, whereas for the LAADF-STEM reconstruction the shape error was found to be $8.0 \%$. Although LAADF-STEM tomography clearly yields superior $3 \mathrm{D}$ information on the location of twin planes, our comparison shows that errors will occur when trying to calculate, for example, the volume of the nanoparticle. Also for the MAADF-STEM reconstruction, the shape error is significant. To overcome this problem, we propose to combine HAADF-STEM and LAADF-STEM reconstructions. In this manner, quantifiable information on the shape of the nanoparticles can be obtained, together with a clear visualization of the twin planes.

To combine the LAADF-STEM and HAADF-STEM reconstructions in a straightforward manner, tilt series were simultaneously acquired using two annular detectors with acquisition angles ranging from 13 to $115 \mathrm{mrad}$ for LAADFSTEM and from 115 to $157 \mathrm{mrad}$ for HAADF-STEM. Because the acquisition was performed simultaneously, the alignment parameters for both series are identical. Next, 3D reconstructions were performed by using the SIRT algorithm. To obtain a better visualization of the position of the twin planes from the LAADF-STEM reconstruction, a manual segmentation was performed. Figure 2 shows a $3 \mathrm{D}$ visualization of the HAADF-
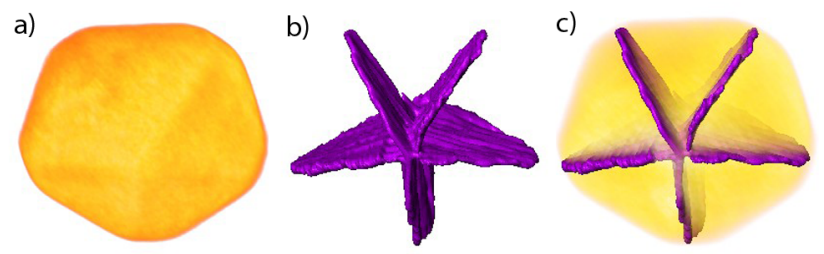

Figure 2. 3D visualization of the HAADF-STEM reconstruction (a) and the twin planes segmented from the LAADF-STEM reconstruction (b) of an $\mathrm{Au}$ decahedron. Both volumes are superimposed to evaluate the position of the twin planes in the correct volume (c).

STEM reconstruction (Figure 2a), as well as the segmented twin planes (Figure 2b). By superimposing both reconstructions (Figure 2c), we are able to simultaneously determine the shape of the decahedron and the position of the twin planes. Animated versions of the reconstruction can be found as Movie S1 in the Supporting Information.

Application toward Mechanistic Studies: Au Structures Grown from a Bimetallic Seed. Two different growth mechanisms have been reported for these pentatwinned nanoparticles. $^{21,35}$ The first pathway involves the nucleation of a twinned seed and its subsequent overgrowth in a layer-bylayer fashion. Alternatively, a single-crystalline seed can develop twin defects during overgrowth, finally resulting in a twinned structure. To gain a better understanding of the growth mechanism, the position of the Au seed inside the final $\mathrm{Au}$ 
nanoparticle and its location with respect to the twins was investigated using the multimode approach as described above.

We recently reported that the growth of pentatwinned $\mathrm{Au}$ nanoparticles of different shapes (decahedra, rods, and bipyramids) from pentatwinned $\mathrm{Au}$ seeds can be achieved, even if the seeds are coated by a thin layer of $\mathrm{Pd}^{31}$ The difference in atomic number between $\mathrm{Au}$ and $\mathrm{Pd}\left(\mathrm{Z}_{\mathrm{Pd}}=46, \mathrm{Z}_{\mathrm{Au}}\right.$ $=79$ ) enabled us to accurately find the position of the $\mathrm{Au} / \mathrm{Pd}$ seed in the final $\mathrm{Au}$ nanoparticle, using HAADF-STEM tomography. In our previous paper, ${ }^{31}$ we confirmed that the presence of Pd did not alter the structure of the seed. We now applied HAADF-STEM tomography to Au bipyramids with an average length of $36.0 \pm 1.2,48.9 \pm 2.5$, and $40.2 \pm 2.1 \mathrm{~nm}$, which were grown from $\mathrm{Au} / \mathrm{Pd}$ seeds with varying $\mathrm{Pd}$ content $(10,20$, and $50 \mathrm{~mol} \%)$, observing a slight increase of the seeds diameter $(6.2 \pm 0.5,6.3 \pm 0.7$, and $6.6 \pm 0.6 \mathrm{~nm}$, respectively). See Figure S2 for details. Orthoslices through the reconstructions are shown in Figure 3. The position of the $\mathrm{Au} / \mathrm{Pd}$ seed
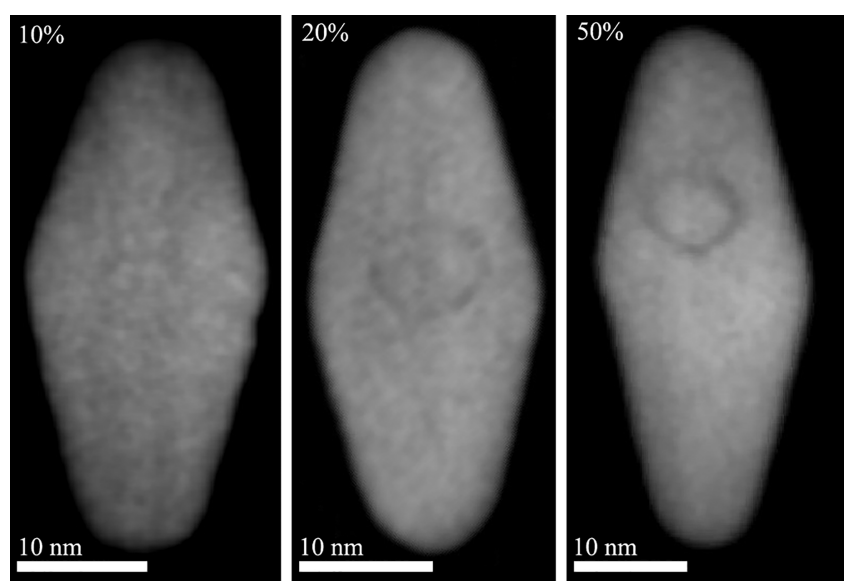

Figure 3. Orthoslices through the HAADF-STEM reconstructions of bipyramids grown from seeds containing different amounts of Pd (10, 20 , and $50 \mathrm{~mol} \%$ ). Depending on the Pd content, the seed is observed either at the longitudinal center or away from it.

can be determined through the lower intensity of the Pd shell, originating from Z-contrast in HAADF-STEM. Although the amount of Pd apparently does not influence the position of the seed in the transversal direction, the seed appears more offcenter in the longitudinal direction when the Pd content was increased. Indeed, for a low amount of Pd $(10 \mathrm{~mol} \%)$ the seed was found to be located at the longitudinal center, for six different particles. For $20 \mathrm{~mol} \% \mathrm{Pd}$, the seed was mostly located at the center ( 7 out of 10 of the investigated particles) and for $50 \mathrm{~mol} \% \mathrm{Pd}$, the seed was mostly displaced toward one end ( 9 out of 10 investigated particles).

The observed longitudinal displacement of the Pd-coated seeds within bipyramids was also reflected in the quality of the samples. Indeed, the relative amount of bipyramids dropped down to $70 \%$ for seeds containing $50 \mathrm{~mol} \% \mathrm{Pd}$, in agreement with a lower quality of the optical response of this sample (Figure S3). Overall, these results confirm that size, composition, and crystallinity of the seeds are critical parameters to ensure homogeneous symmetry breaking and high-quality anisotropic nanoparticles. ${ }^{3}$

To investigate the position of the seed with respect to the twin planes (transversal seeds displacement), multimode tomography was performed. Three different morphologies were investigated: decahedra $(27.5 \pm 0.9 \mathrm{~nm})$, nanorods $(51.1$ $\pm 2.1 \mathrm{~nm})$, and bipyramids $(48.9 \pm 2.5 \mathrm{~nm})$, grown on the seeds containing $20 \mathrm{~mol} \%$ of Pd. For each sample, tilt series were simultaneously acquired using HAADF-STEM and LAADF-STEM over a tilt range from $-72^{\circ}$ to $+70^{\circ}$, with a tilt increment of $2^{\circ}$. Orthoslices through the HAADF-STEM reconstructions of the different pentatwinned structures are shown in the top row of Figure 4. The Pd shell around the Au

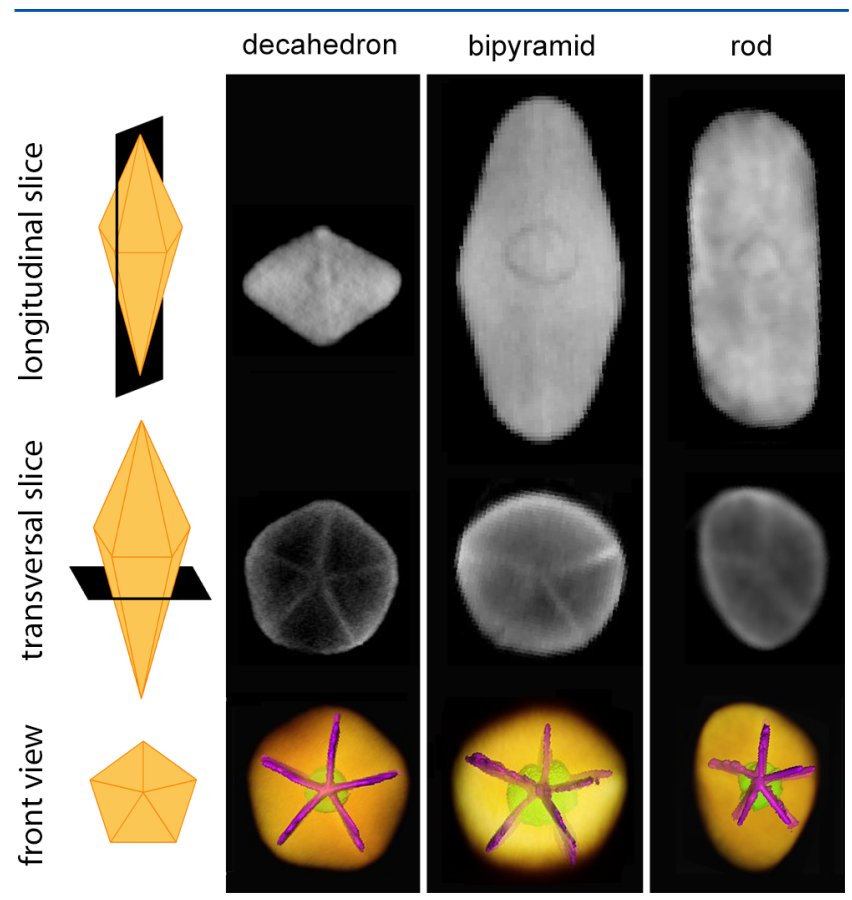

Figure 4. Top row: Longitudional orthoslices through the HAADFSTEM reconstruction of a decahedron, a bipyramid and a nanorod grown from $\mathrm{Au} / \mathrm{Pd}$ seeds (Pd $20 \mathrm{~mol} \%)$. Middle row: Transversal orthoslices through the LAADF-STEM reconstruction, from which the twins can be segmented. Bottom row: The segmented seed and twin planes superimposed with the HAADF-STEM reconstruction.

seeds can be clearly seen. The twin planes can be observed from orthogonal slices through the LAADF-STEM reconstructions (middle row in Figure 4). Next, manual segmentation of the twins was performed for the LAADF-STEM reconstructions. By superimposing the HAADF-STEM and LAADFSTEM reconstructions (bottom row of Figure 4), we were able to determine the shape of all pentatwinned particles, as well as the position of the seed with respect to the twin planes. An animated version of the $3 \mathrm{D}$ reconstructions of the decahedron in Figure 4 is presented as Supporting Information, Movie S2. These results enabled us to confirm that the overgrown $\mathrm{Au}$ particles are invariably pentatwinned and that the seed is located at the connection point of the five twins.

As a confirmation for the epitaxial relationship between the $\mathrm{Au} / \mathrm{Pd}$ seed and the Au shell, high resolution HAADF-STEM images can be acquired along the long axis of the structures. However, for nanorods and bipyramids, such an analysis is hampered by the relatively large thickness of the investigated structures. An example is presented in Figure 5a, where a high resolution HAADF-STEM image of a bipyramid oriented along a [110] axis is presented. Due to the large amount of $\mathrm{Au}$ as compared to $\mathrm{Pd}$, the contrast is not sufficient to identify the position of the seed in a reliable manner. To overcome this limitation, the particles were deposited on a Si substrate, and a 


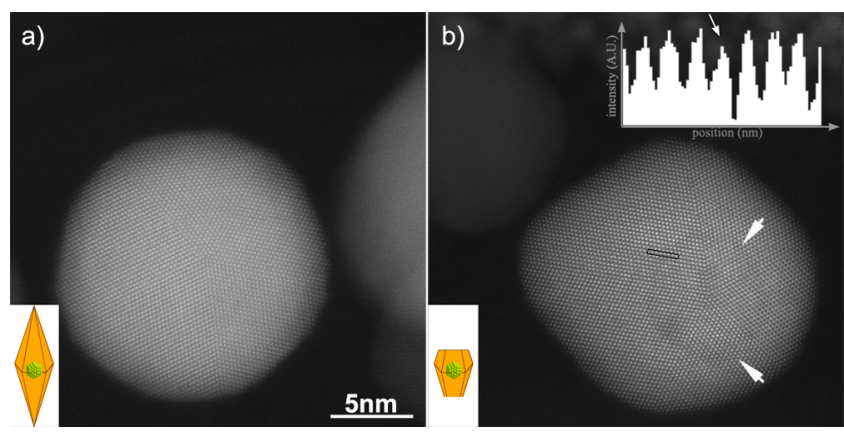

Figure 5. High resolution HAADF-STEM images of Au bipyramids oriented along a $\langle 110\rangle$ zone axis, in its original shape (a) and after FIB milling (b). Schematic representations of the particles are shown as insets. The position of the Pd layer is indicated by the two white arrows in b. A line profile along the black rectangle, shown as inset, shows a clear decrease in intensity at the position of the Pd layer.

cross-section cut of the sample, with a thickness of approximately $20 \mathrm{~nm}$ was obtained using focused ion beam (FIB) milling. Due to the smaller thickness of the resulting specimen, the Pd layer could be observed in high resolution (Figure $5 b$ ), clearly showing that the growth of $\mathrm{Au}$ on the bimetallic seed is epitaxial.

Application toward Mechanistic Studies: Au Structures Grown from a Pd Seed. To understand the effect of size and composition of the seeds on their localization within the final particles, we applied the multimode tomography technique to analyze pentatwinned Au bipyramids grown from pure Pd seeds, with a length of $73.9 \pm 3.9 \mathrm{~nm}$. First, we noticed that the thermal treatment of as-prepared pure Pd seeds led to nanoparticles with a smaller diameter $(2 \mathrm{~nm})$ than that of pure $\mathrm{Au}$ seeds (6 $\mathrm{nm}$ upon thermal treatment $\left.{ }^{29}\right)$. Such a size limitation in Pd seeds may hinder the formation of twin planes, resulting in a low yield of bipyramids (30\%, see Figure S4).

Tilt series were acquired simultaneously in HAADF-STEM and LAADF-STEM modes, within a tilt range from $-74^{\circ}$ to $+72^{\circ}$ and a tilt increment of $2^{\circ}$. The Pd seed and the twin planes were manually segmented from the HAADF-STEM and LAADF-STEM reconstructions, respectively, and then superimposed to visualize the position of the seed with respect to the twin planes. In this case, the seed can be either positioned at the connection point between the twin planes (Figure 6a), which is similar to what we observed in the particles described above, or next to that connection point (Figure 6b), thereby suggesting that twinning occurred in this case during
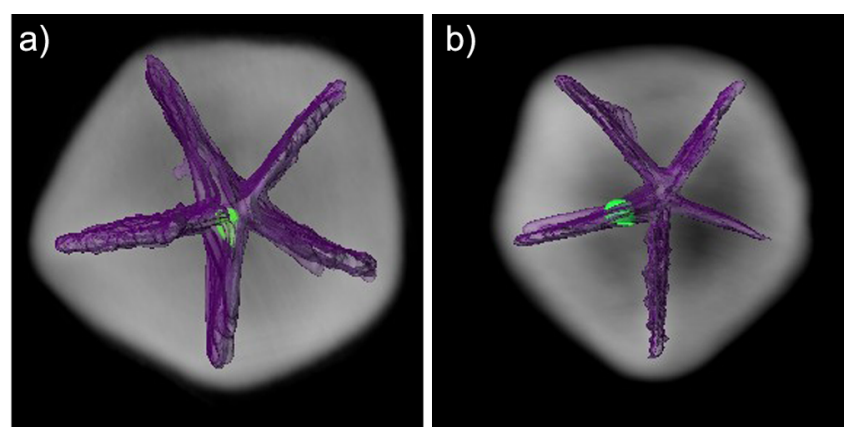

Figure 6. The position of the $\mathrm{Pd}$ seed inside $\mathrm{Au}$ bipyramids was determined using multimode tomography. The seed is located either at the connection point of the five twin planes (a) or next to it (b). overgrowth, prior to the actual development of the bipyramidal shape. Due to the small size of the seeds, high resolution HAADF-STEM imaging of samples prepared by FIB milling as described above was particularly useful in this case. Furthermore, due to the low contrast of the Pd seed in the HAADF-STEM images, EDS measurements were performed to confirm its presence and location. These results are shown in the Supporting Information, Figures S5 and S6. In total, only 8 out of the 17 investigated bipyramids showed a seed located at the connection point of the twin planes.

Overall, these results again confirm that the formation of bipyramids (and in general anisotropic nanoparticles) is strongly related to features of the seeds such as size, composition, and crystallinity. In this context, multimode tomography characterization becomes an extremely valuable tool in understanding and gaining further control over the growth mechanisms. For example, our results provide evidence of growth pathway alteration depending on seed composition (Pt vs Au@Pt), as indicated by longitudinal and transversal displacement of the seed position, even though the final particles have a similar bipyramidal morphology. On the contrary, if the seed features (size, crystallinity) overcome a certain threshold, the system cannot support symmetry breaking and results in isotropic growth, with a corresponding decrease in bipyramids population. We are aware that a further effort is required to correlate statistically meaningful data on the macroscopic level with the information obtained on the singleparticle level. However, the implementation of multimode electron tomography will help us understand how seed features translate into the final nanoparticle morphology.

\section{CONCLUSIONS}

In this work, we demonstrated the potential of combined HAADF-STEM and LAADF-STEM tomography to identify twin planes in Au nanostructures. Tilt series were acquired in a dose-efficient manner by simultaneously collecting images using two different annular detectors. After a stringent segmentation, both reconstructions were superimposed to reveal the positions of twin planes in the nanoparticles. As an application, we used this approach to investigate the relation between twins in anisotropic $\mathrm{Au}$ nanoparticles and the seed from which their growth was initiated. The results were further confirmed by high resolution HAADF-STEM imaging on particles from samples prepared by FIB milling. It should be noted that the methodology proposed here is generally applicable to a broad range of homogeneous and heterogeneous nanoparticles.

\section{ASSOCIATED CONTENT}

\section{Supporting Information}

The Supporting Information is available free of charge on the ACS Publications website at DOI: 10.1021/acs.jpcc.7b12379.

Schematic diagram of the experimental setup, animated versions of the tomographic reconstructions, UV-visNIR characterization of the nanoparticles, EDX spectroscopy of the Au bipyramids grown from a pure $\mathrm{Pd}$ seed are supplied (PDF).

Movie S1 (MPG).

Movie S2 (MPG).

\section{AUTHOR INFORMATION}

\section{Corresponding Author}

*E-mail: sara.bals@uantwerpen.be. 


\section{ORCID}

Marek Grzelczak: 0000-0002-3458-8450

Luis M. Liz-Marzán: 0000-0002-6647-1353

Sara Bals: 0000-0002-4249-8017

\section{Notes}

The authors declare no competing financial interest.

\section{ACKNOWLEDGMENTS}

S.B. and N.W. acknowledge funding from the European Research Council under the Seventh Framework Program (FP7), ERC Grant No. 335078 COLOURATOM. S.B. and T.A. acknowledge financial support from the Research Foundation Flanders (FWO, Belgium) through project fundings (G.0369.15N and G.0218.14N) and a postdoctoral research grant to T.A. L.M.L.-M. and M.G. acknowledge funding from the Spanish Ministerio de Economía $y$ Competitividad (Grant MAT2013-46101-R). L.M.L.-M. and S.B. acknowledge funding from the European Commission (Grant EUSMI 731019).

\section{REFERENCES}

(1) Tian, Z. Q.; Ren, B.; Wu, D. Y. Surface-Enhanced Raman Scattering: From Noble to Transition Metals and from Rough Surfaces to Ordered Nanostructures. J. Phys. Chem. B 2002, 106, 9463-9483.

(2) Pelton, M.; Aizpurua, J.; Bryant, G. Metal-Nanoparticle Plasmonics. Laser Photonics Rev. 2008, 2, 136-159.

(3) Haes, A. J.; Stuart, D. A.; Nie, S.; Van Duyne, R. P. Using Solution-Phase Nanoparticles, Surface-Confined Nanoparticle Arrays and Single Nanoparticles as Biological Sensing Platforms. J. Fluoresc. 2004, 14, 355-367.

(4) Langille, M. R.; Personick, M. L.; Zhang, J.; Mirkin, C. A. Defining Rules for the Shape Evolution of Gold Nanoparticles. J. Am. Chem. Soc. 2012, 134, 14542-14554.

(5) Lu, X.; Rycenga, M.; Skrabalak, S. E.; Wiley, B.; Xia, Y. Chemical Synthesis of Novel Plasmonic Nanoparticles. Annu. Rev. Phys. Chem. 2009, 60, 167-192.

(6) Liz-Marzán, L. M.; Grzelczak, M. Growing Anisotropic Crystals at the Nanoscale. Science 2017, 356, 1120-1121.

(7) Scarabelli, L.; Sánchez-Iglesias, A.; Pérez-Juste, J.; Liz-Marzán, L. M. A "Tips and Tricks" Practical Guide to the Synthesis of Gold Nanorods. J. Phys. Chem. Lett. 2015, 6, 4270-4279.

(8) Midgley, P. A.; Weyland, M. 3D Electron Microscopy in the Physical Sciences: The Development of Z-Contrast and EFTEM Tomography. Ultramicroscopy 2003, 96, 413-431.

(9) Midgley, P. A.; Dunin-Borkowski, R. E. Electron Tomography and Holography in Materials Science. Nat. Mater. 2009, 8, 271-280.

(10) Ersen, O.; Florea, I.; Hirlimann, C.; Pham-Huu, C. Exploring Nanomaterials with 3D Electron Microscopy. Mater. Today 2015, 18, 395-408.

(11) Möbus, G.; Doole, R. C.; Inkson, B. J. Spectroscopic Electron Tomography. Ultramicroscopy 2003, 96, 433-451.

(12) Liakakos, N.; Gatel, C.; Blon, T.; Altantzis, T.; Lentijo-Mozo, S.; Garcia-Marcelot, C.; Lacroix, L. M.; Respaud, M.; Bals, S.; Van Tendeloo, G.; et al. Co-Fe Nanodumbbells: Synthesis, Structure, and Magnetic Properties. Nano Lett. 2014, 14, 2747-2754.

(13) Goris, B.; Meledina, M.; Turner, S.; Zhong, Z.; Batenburg, K. J.; Bals, S. Three Dimensional Mapping of Fe Dopants in Ceria Nanocrystals Using Direct Spectroscopic Electron Tomography. Ultramicroscopy 2016, 171, 55-62.

(14) Goubet, N.; Yan, C.; Polli, D.; Portalès, H.; Arfaoui, I.; Cerullo, G.; Pileni, M. P. Modulating Physical Properties of Isolated and SelfAssembled Nanocrystals through Change in Nanocrystallinity. Nano Lett. 2013, 13, 504-508.

(15) Louis, C., Pluchery, O., Eds. Gold Nanoparticles for Physics, Chemistry and Biology; Imperial College Press, 2012; Vol. XXXIII.
(16) Novo, C.; Funston, A. M.; Mulvaney, P. Direct Observation of Chemical Reactions on Single Gold Nanocrystals Using Surface Plasmon Spectroscopy. Nat. Nanotechnol. 2008, 3, 598-602.

(17) Walker, A. M.; Slater, B.; Gale, J. D.; Wright, K. Predicting the Structure of Screw Dislocations in Nanoporous Materials. Nat. Mater. 2004, 3, 715-720.

(18) Liu, M.; Jing, D.; Zhou, Z.; Guo, L. Twin-Induced OneDimensional Homojunctions Yield High Quantum Efficiency for Solar Hydrogen Generation. Nat. Commun. 2013, 4, 2278-2285.

(19) Grzelczak, M.; Pérez-Juste, J.; Mulvaney, P.; Liz-Marzán, L. M. Shape Control in Gold Nanoparticle Synthesis. Chem. Soc. Rev. 2008, 37, 1783.

(20) Kumar, S.; Yang, H.; Zou, S. Seed-Mediated Growth of Uniform Gold Nanoparticle Arrays. J. Phys. Chem. C 2007, 111, 12933-12938.

(21) Skrabalak, S. E.; Xia, Y. Pushing Nanocrystal Synthesis toward Nanomanufacturing. ACS Nano 2009, 3, 10-15.

(22) Walsh, M. J.; Barrow, S. J.; Tong, W.; Funston, A. M.; Etheridge, J. Symmetry Breaking and Silver in Gold Nanorod Growth. ACS Nano 2015, 9, 715-724.

(23) Langille, M. R.; Zhang, J.; Personick, M. L.; Li, S.; Mirkin, C. A. Stepwise Evolution of Spherical Seeds into 20-Fold Twinned Icosahedra. Science 2012, 337, 954-957.

(24) Chen, C.-C.; Zhu, C.; White, E. R.; Chiu, C.-Y.; Scott, M. C.; Regan, B. C.; Marks, L. D.; Huang, Y.; Miao, J. Three-Dimensional Imaging of Dislocations in a Nanoparticle at Atomic Resolution. Nature 2013, 496, 74-77.

(25) Goris, B.; De Beenhouwer, J.; De Backer, A.; Zanaga, D.; Batenburg, K. J.; Sánchez-Iglesias, A.; Liz-Marzán, L. M.; Van Aert, S.; Bals, S.; Sijbers, J.; et al. Measuring Lattice Strain in Three Dimensions through Electron Microscopy. Nano Lett. 2015, 15, 6996-7001.

(26) Kacher, J.; Robertson, I. M. In Situ and Tomographic Analysis of Dislocation/grain Boundary Interactions in $\alpha$-Titanium. Philos. Mag. 2014, 94, 814-829.

(27) Barnard, J. S.; Eggeman, A. S.; Sharp, J.; White, T. A.; Midgley, P. A. Dislocation Electron Tomography and Precession Electron Diffraction - Minimising the Effects of Dynamical Interactions in Real and Reciprocal Space. Philos. Mag. 2010, 90, 4711-4730.

(28) Tanaka, M.; Higashida, K.; Kaneko, K.; Hata, S.; Mitsuhara, M. Crack Tip Dislocations Revealed by Electron Tomography in Silicon Single Crystal. Scr. Mater. 2008, 59, 901-904.

(29) Eggeman, A. S.; Krakow, R.; Midgley, P. A. Scanning Precession Electron Tomography for Three-Dimensional Nanoscale Orientation Imaging and Crystallographic Analysis. Nat. Commun. 2015, 6, 72677273.

(30) Midgley, P. A.; Weyland, M.; Thomas, J. M.; Johnson, B. F. G. Z-Contrast Tomography: A Technique Inthree-Dimensional Nanostructural Analysis Based on Rutherfordscattering. Chem. Commun. 2001, 907-908.

(31) Sánchez-Iglesias, A.; Winckelmans, N.; Altantzis, T.; Bals, S.; Grzelczak, M.; Liz-Marzán, L. M. High-Yield Seeded Growth of Monodisperse Pentatwinned Gold Nanoparticles through Thermally Induced Seed Twinning. J. Am. Chem. Soc. 2017, 139, 107-110.

(32) Martinez, G. T.; Jones, L.; De Backer, A.; Beche, A.; Verbeeck, J.; Van Aert, S.; Nellist, P. D. Quantitative STEM Normalisation: The Importance of the Electron Flux. Ultramicroscopy 2015, 159, 46-58.

(33) Schoenmakers, R. H. M.; Perquin, R. A.; Fliervoet, T. F.; Voorhout, W.; Schirmacher, H. New Software for High Resolution, High Throughput Electron Tomography. Microsc. Anal. 2005, 19, 5-6.

(34) Palenstijn, W. J.; Batenburg, K. J.; Sijbers, J. Performance Improvements for Iterative Electron Tomography Reconstruction Using Graphics Processing Units (GPUs). J. Struct. Biol. 2011, 176, 250-253.

(35) van Aarle, W.; Palenstijn, W. J.; De Beenhouwer, J.; Altantzis, T.; Bals, S.; Batenburg, K. J.; Sijbers, J. The ASTRA Toolbox: A Platform for Advanced Algorithm Development in Electron Tomography. Ultramicroscopy 2015, 157, 35-47.

(36) Bladt, E.; Pelt, D. M.; Bals, S.; Batenburg, K. J. Electron Tomography Based on Highly Limited Data Using a Neural Network Reconstruction Technique. Ultramicroscopy 2015, 158, 81-88. 
(37) Tong, W.; Walsh, M. J.; Mulvaney, P.; Etheridge, J.; Funston, A. M. Control of Symmetry Breaking Size and Aspect Ratio in Gold Nanorods: Underlying Role of Silver Nitrate. J. Phys. Chem. C 2017, 121, 3549-3559. 